\title{
Dynamic Range Analysis of the Phase Generated Carrier Demodulation Technique
}

\author{
M. J. Plotnikov, A. V. Kulikov, V. E. Strigalev, and I. K. Meshkovsky \\ Saint Petersburg National Research University of Information Technologies, Mechanics and Optics, \\ Kronverkskiy Prospekt 49, Saint Petersburg 197101, Russia \\ Correspondence should be addressed to M. J. Plotnikov; plotnikov-michael@yandex.ru
}

Received 29 November 2013; Revised 15 February 2014; Accepted 16 February 2014; Published 17 March 2014

Academic Editor: Joseph Rosen

Copyright (C) 2014 M. J. Plotnikov et al. This is an open access article distributed under the Creative Commons Attribution License, which permits unrestricted use, distribution, and reproduction in any medium, provided the original work is properly cited.

The dependence of the dynamic range of the phase generated carrier (PGC) technique on low-pass filters passbands is investigated using a simulation model. A nonlinear character of this dependence, which could lead to dynamic range limitations or measurement uncertainty, is presented for the first time. A detailed theoretical analysis is provided to verify the simulation results and these results are consistent with performed calculations. The method for the calculation of low-pass filters passbands according to the required dynamic range upper limit is proposed.

\section{Introduction}

Fiber optic interferometric sensors have been intensively developed for the past few decades. Fiber-optic interferometers are used as a sensitive element in fiber optic gyroscopes, hydrophones, and so forth [1]. As a result, many special data acquisition methods, digital signal processing algorithms, and demodulation techniques have been invented to recover measured phase signals. One of the most popular demodulation techniques is phase generated carrier (PGC) demodulation technique [2].

This technique has been used in many applications such as hydroacoustics [3-5], vibration [6], and pipeline leakage detection [7], and its main properties have been thoroughly studied [8-10].

However, implementation methods of demodulation techniques have changed with the advent of high performance field programmable gate arrays (FPGAs). FPGAs allow realizing complex real-time digital signal processing algorithms, and therefore they are ideally suitable for the multichannel PGC technique implementation to demodulate signals from fiber-optic sensor arrays [11]. Unfortunately, the usage of FPGAs for such purposes has got some problems caused by sampling frequency restrictions and finite passbands of digital schemes.
This paper is devoted to the detailed analysis of the PGC technique taking into account its practical implementation problems and digital design restrictions. The dependence of the dynamic range of the PGC technique on low-pass filters passbands is investigated for the first time and the nonlinear character of this dependence is shown and explained.

\section{Simulation}

The PGC demodulation scheme is presented in Figure 1 [11]. Its functional model was implemented using MATLAB to investigate dependences of output signals on various parameters. Filter Design and Analysis Tool (FDATool) was used for generating necessary finite impulse response digital filters.

Several parameters were constants in this model:

(i) the duration of simulated signals was 1 second;

(ii) the carrier frequency was set to $10 \mathrm{kHz}$;

(iii) the sampling frequency of the model was $100 \mathrm{kHz}$.

Low-pass filters passbands were set to $500 \mathrm{~Hz}$ hence the input signal at the frequency $500 \mathrm{~Hz}$ passed through these filters without attenuation.

Results of performed simulations are shown in Figure 2. 


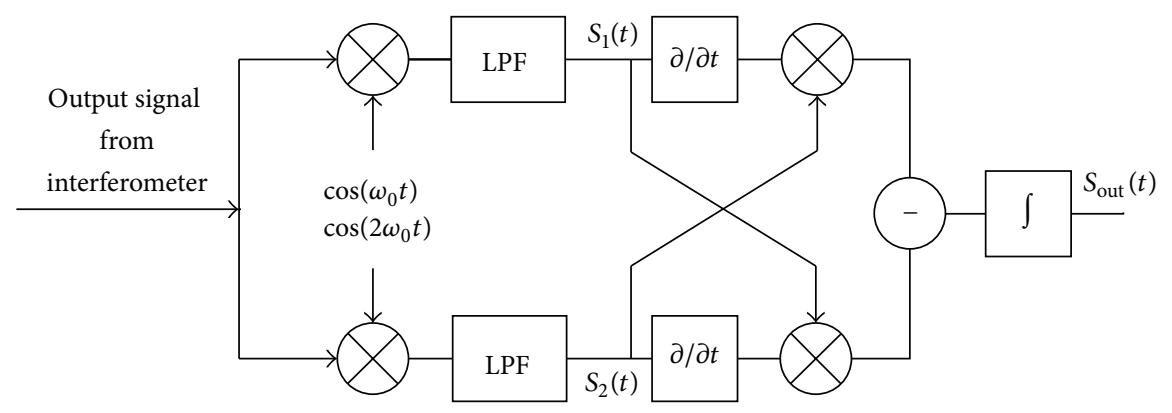

Figure 1: PGC demodulation scheme.

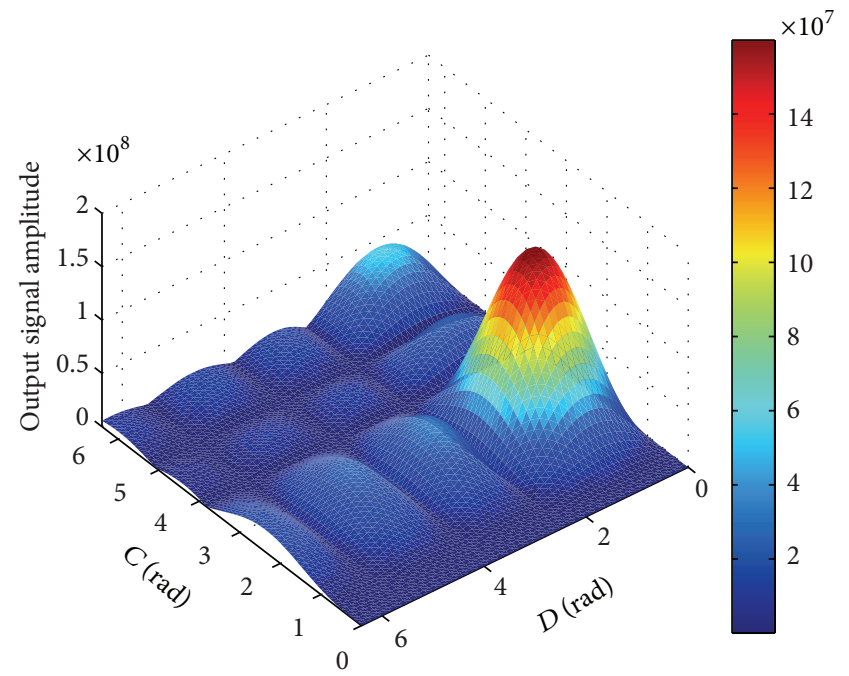

FIGURE 2: Simulation results of the PGC demodulation scheme: dependencies of the output signal on the measured phase signal amplitude $(D)$ and the carrier modulation depth $(C)$.

During this simulation the amplitude of the measured phase signal (at the frequency $500 \mathrm{~Hz}$ ) and the carrier modulation depth were changing from 0 to $6.28 \mathrm{rad}$.

According to obtained results (see Figure 2), the output signal from the PGC demodulation scheme depends nonlinearly on both the measured phase signal amplitude $(D)$ and the carrier modulation depth $(C)$. To get a detailed explanation of these results, both dependencies were analyzed separately.

\section{Analysis}

The demodulated result of the PGC scheme (see Figure 1) for small signal amplitudes and small signal frequencies is expressed as $[11,12]$

$$
\begin{gathered}
S_{\text {out }}(t)=B^{2} J_{1}(C) J_{2}(C) \varphi(t), \\
\varphi(t)=D \cos \omega t+\varphi_{0},
\end{gathered}
$$

where $B$ depends on the input optical power and the mixing efficiency of the interferometer, $C$ is carrier modulation depth, $J_{i}(C)$ with $i=1,2, \ldots$, are the first-order and secondorder Bessel functions with $C, D$ is the measured phase signal amplitude, $\omega$ is the angular frequency of the measured phase signal, and $\varphi_{0}$ is the initial phase of the interferometer.

As shown in Figure 2, the amplitude dependence of the output signal on the carrier modulation depth is directly proportional to the result of multiplication $J_{1}(C) J_{2}(C)$. This result is consistent with (1). Furthermore, it can be seen that the optimal value of the carrier modulation depth, which provides the local extreme value of $J_{1}(C) J_{2}(C)$ and corresponds to the maximum amplitude of the output signal, is $C \approx 2.37 \mathrm{rad}$ [12].

According to (1), the amplitude dependence of the output signal from the PGC demodulation scheme is a linear function for small input signal amplitudes; that is, the amplitude of the output signal is directly proportional to the amplitude of measured phase signals. However, obtained results show that this dependence is nonlinear for large input signal amplitudes (see Figure 2).

In order to explain obtained results, signals after low-pass filters in the PGC demodulation scheme (see Figure 1) should be expressed in detail as

$$
\begin{aligned}
& S_{1}(t) \\
& =-B J_{1}(C) \\
& \quad \times\left(\cos \varphi_{0}\left[2 \sum_{k=0}^{\infty}(-1)^{k} J_{2 k+1}(D) \cos [(2 k+1) \omega t]\right]\right. \\
& \left.\quad+\sin \varphi_{0}\left[J_{0}(D)+2 \sum_{k=1}^{\infty}(-1)^{k} J_{2 k}(D) \cos (2 k \omega t)\right]\right), \\
& S_{2}(t) \quad-\quad\left(\cos \varphi_{0}\left[J_{0}(D)+2 \sum_{k=1}^{\infty}(-1)^{k} J_{2 k}(D) \cos (2 k \omega t)\right]\right. \\
& \left.\quad-\sin \varphi_{0}\left[2 \sum_{k=0}^{\infty}(-1)^{k} J_{2 k+1}(D) \cos [(2 k+1) \omega t]\right]\right) .
\end{aligned}
$$


According to (3), signals $S_{1}(t)$ and $S_{2}(t)$ have got infinite spectrum, which consists of harmonics. They are proportional to $J_{n}(D)$, where $n \geq 1$.

But during the practical implementation of the digital PGC scheme it is impossible to provide infinite passbands of digital low-pass filters. Anyway, all passbands will be limited by the digital scheme sampling frequency. Therefore, some high-order harmonics of signals $S_{1}(t)$ and $S_{2}(t)$ might be filtered out.

To determine the output signal dependence on the amplitude of the measured phase signal, further PGC technique operations on signals $S_{1}(t)$ and $S_{2}(t)$ should be performed according to Figure 1 for several cases: when signals $S_{1}(t)$ and $S_{2}(t)$ contain only the first harmonic of the measured phase signal, two harmonics, three harmonics, and so forth. These cases will be matched to appropriate low-pass filters passbands.

To simplify these calculations, low-pass filters are supposed to be ideal and thus all filtered out harmonics will not be present in filtered signals. The initial phase of the interferometer is considered to be constant.

Then, in the simplest case, when low-pass filters passbands are equal to the fundamental frequency of the measured phase signal (i.e., low-pass filters passbands include the first harmonic of the measured phase signal and the input signal at the frequency of this harmonic could pass through low-pass filters without attenuation), signals (3) can be represented as

$$
\begin{aligned}
S_{1}(t)= & -2 B J_{1}(C) J_{1}(D) \cos \omega t \cos \varphi_{0} \\
& -B J_{1}(C) J_{0}(D) \sin \varphi_{0}, \\
S_{2}(t)= & 2 B J_{2}(C) J_{1}(D) \cos \omega t \sin \varphi_{0} \\
& -B J_{2}(C) J_{0}(D) \cos \varphi_{0} .
\end{aligned}
$$

Derivatives of (4) and (5) can be expressed as

$$
\begin{gathered}
\frac{\partial S_{1}(t)}{\partial t}=2 B J_{1}(C) J_{1}(D) \omega \sin \omega t \cos \varphi_{0}, \\
\frac{\partial S_{2}(t)}{\partial t}=-2 B J_{2}(C) J_{1}(D) \omega \sin \omega t \sin \varphi_{0} .
\end{gathered}
$$

Then cross-multiplication between signals (4) and (7), (5), and (6) yields

$$
\begin{aligned}
S_{1}( & t) \\
= & 4 B^{2} J_{1}(C) J_{2}(C) J_{1}^{2}(D) \omega \sin \omega t \cos \omega t \sin \varphi_{0} \cos \varphi_{0} \\
& +2 B^{2} J_{1}(C) J_{2}(C) J_{0}(D) J_{1}(D) \omega \sin \omega t \sin ^{2} \varphi_{0}, \\
S_{2}(t) & \frac{\partial S_{1}(t)}{\partial t} \\
= & 4 B^{2} J_{1}(C) J_{2}(C) J_{1}^{2}(D) \omega \sin \omega t \cos \omega t \sin \varphi_{0} \cos \varphi_{0} \\
& -2 B^{2} J_{1}(C) J_{2}(C) J_{0}(D) J_{1}(D) \omega \sin \omega t \cos ^{2} \varphi_{0} .
\end{aligned}
$$

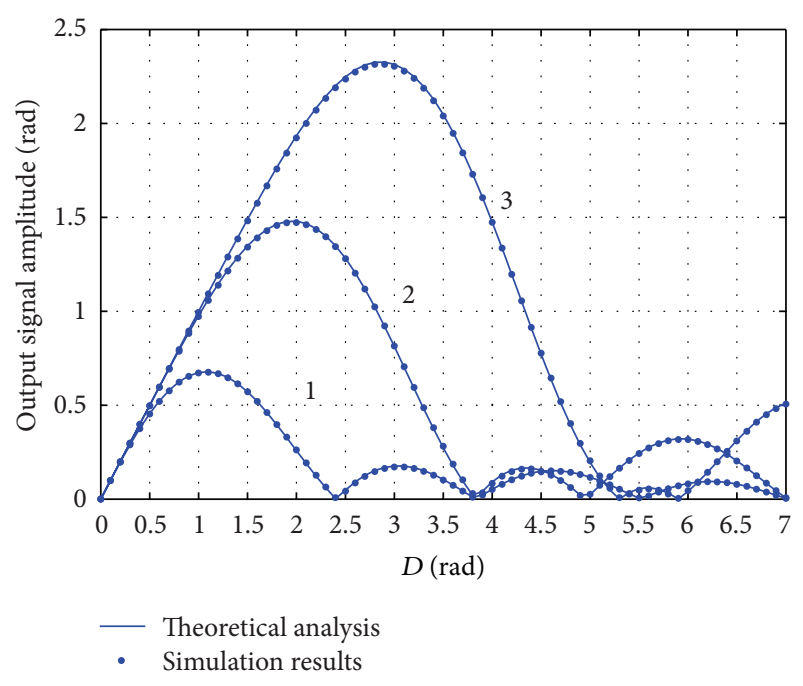

FIGURE 3: Theoretical and simulation results: output signal amplitude dependencies on the measured phase signal amplitude $(D)$ for various low-pass filter passbands: $1-500 \mathrm{~Hz} ; 2-1000 \mathrm{~Hz} ; 3-$ $1500 \mathrm{~Hz}$.

Subtracting the signal (8) from (9) gives

$$
\begin{aligned}
S_{2} & (t) \frac{\partial S_{1}(t)}{\partial t}-S_{1}(t) \frac{\partial S_{2}(t)}{\partial t} \\
& =-2 B^{2} J_{1}(C) J_{2}(C) J_{0}(D) J_{1}(D) \omega \sin \omega t .
\end{aligned}
$$

After the integration, the output signal can be expressed as

$$
S_{\text {out }}(t)=2 B^{2} J_{1}(C) J_{2}(C) J_{0}(D) J_{1}(D) \cos \omega t .
$$

Performing the same mathematical manipulations with signals $S_{1}(t)$ and $S_{2}(t)$ according to the PGC demodulation scheme (see Figure 1) in cases of wider low-pass filters passbands, the final equation of the output signal amplitude dependence on the input signal amplitude at the fundamental input signal frequency can be obtained:

$$
S_{\text {out }}(t)=2 B^{2} J_{1}(C) J_{2}(C) \sum_{k=0}^{N-1}(2 k+1) J_{k}(D) J_{k+1}(D) \cos \omega t
$$

where $N$ is equal to the number of useful harmonics of the measured phase signal that are arranged in low-pass filters passbands.

To verify the obtained result (12), the special simulation was performed with various low-pass filter passbands: $500 \mathrm{~Hz}, 1000 \mathrm{~Hz}$, and $1500 \mathrm{~Hz}$. Corresponding theoretical dependences (according to (12)) and simulation results (normalized according to the dependence on $B$ and $C$ ) are presented in Figure 3.

The plot in Figure 3 represents the output signal amplitude at the fundamental input signal frequency $500 \mathrm{~Hz}$. According to Figure 3, the theoretical amplitude dependence of the output signal on the measured phase signal amplitude 


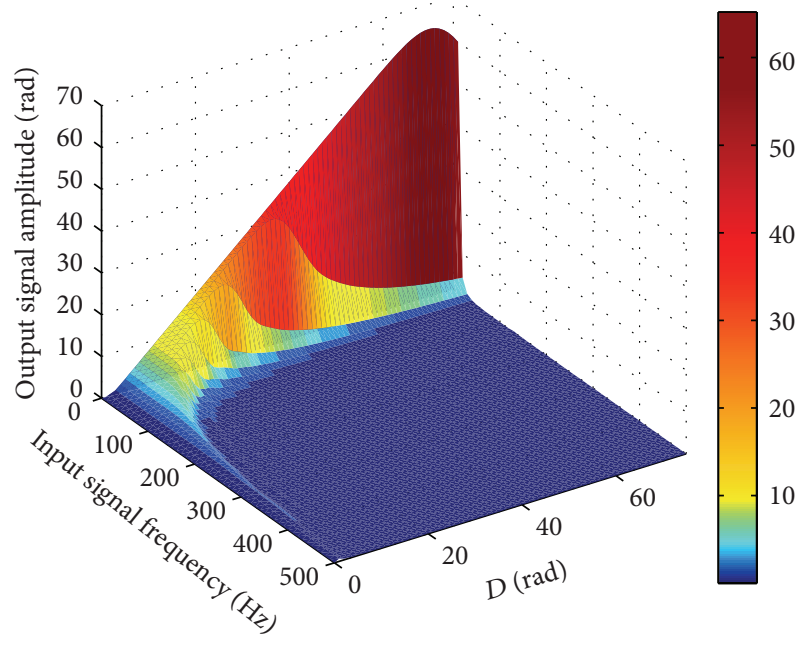

FIGURE 4: Simulation results: the dynamic range of the PGC demodulation scheme increases with the decrease in the input signal frequency (low-pass filter passbands: $500 \mathrm{~Hz}$ ).

(see (12)) matches simulation results. Therefore, the output signal amplitude of the PGC scheme depends nonlinearly on the measured phase signal amplitude (for large input signal amplitudes) due to restrictions of low-pass filters passbands. It could lead to dynamic range limitations or measurement uncertainty of input phase signals.

This dependence is of significant interest in terms of the practical implementation of the digital PGC technique, because it determines the dynamic range upper limit of the demodulation scheme. The wider the low-pass filters passbands are, the higher the dynamic range is.

It should be noted that the demodulated signal is generally contaminated by high-order harmonics and their amplitude dependencies on the measured phase signal amplitude are also nonlinear. However, a detailed description of their exact behavior is beyond the scope of this paper.

There is one more important result of low-pass filters passbands restrictions, which should be taken into account during the PGC technique implementation. It is the dependence of the dynamic range on the input signal frequency. This dependence (normalized according to the dependence on $B$ and $C$ ) is presented in Figure 4. It should be noted that the dependence on $C$ is known (see (1) and (12)) and the output signal might be corrected for this dependence by taking into account the once measured value of $C$ in a real application.

As shown in Figure 4, the dynamic range of the PGC scheme increases with the decrease in the input signal frequency. It can be explained by increasing the amount of useful harmonics of the input signal, which are arranged in fixed low-pass filter passbands. This result is consistent with the dynamic range analysis [13].

In real demodulation schemes the dynamic range upper limit is determined by the required maximum amplitude of the measured phase signal. Therefore, low-pass filters passbands should be calculated according to this maximum amplitude using (12) during the PGC technique practical implementation.

\section{Conclusion}

In the present work it was shown for the first time that the dependence of the dynamic range of the PGC technique on low-pass filters passbands is nonlinear for large input signal amplitudes because of low-pass filters passbands restrictions. The nonlinear character of this dependence could cause dynamic range limitations or measurement uncertainty of input phase signals (see (12) and Figure 3).

According to obtained results, the additional calculation of low-pass filters passbands should be performed in accordance with requirements to the amplitude dependence of the output signal and the necessary dynamic range of the PGC demodulation scheme.

\section{Conflict of Interests}

The authors declare that there is no conflict of interests regarding the publication of this paper.

\section{Acknowledgments}

This work was done in NRU ITMO and supported by the Ministry of Education and Science of the Russian Federation under Project 02.G25.31.0044.

\section{References}

[1] S. Yin, P. B. Ruffin, and F. T. S. Yu, Fiber Optic Sensors, CRC Press Taylor \& Francis Group, 2nd edition, 2008.

[2] A. Dandridge, A. B. Tveten, and T. G. Gialloronzi, "Homodyne demodulation scheme for fiber optic sensors using phase generated carrier," IEEE Journal of Quantum Electronics, vol. 18, no. 10, pp. 1647-1653, 1982.

[3] R. Li, X. Wang, J. Huang, and H. Gu, "Phase generated carrier technique for fiber laser hydrophone," in International Symposium on Photoelectronic Detection and Imaging 2013: Fiber Optic Sensors and Optical Coherence Tomography, vol. 8914 of Proceedings of SPIE, pp. 1-5, 2013.

[4] Z. Meng, Y. Hu, M. Ni et al., "Development of a 32-element fibre optic hydrophone system," in Fiber Optic Sensor Technology and Applications III, vol. 5589 of Proceedings of SPIE, pp. 114-119, October 2004.

[5] A. D. Kersey, "A review of recent developments in fiber optic sensor technology," Optical Fiber Technology, vol. 2, no. 3, pp. 291-317, 1996.

[6] Y. Li, Z. Liu, Y. Liu, L. Ma, Z. Tan, and S. Jian, "Interferometric vibration sensor using phase-generated carrier method," Applied Optics, vol. 52, no. 25, pp. 6359-6363, 2013.

[7] C. He, L. Hang, and B. Wu, "Application of homodyne demodulation system in fiber optic sensors using phase generated carrier based on LabVIEW in pipeline leakage detection," in 2nd International Symposium on Advanced Optical Manufacturing and Testing Technologies: Optical Test and Measurement Technology and Equipment, vol. 6150 of Proceedings of SPIE, pp. 1-6, November 2006. 
[8] K. Wu, Z. Min, and Y. Liao, "Signal dependence of the phasegenerated carrier method," Optical Engineering, vol. 46, no. 10, Article ID 105602, 5 pages, 2007.

[9] S. Varzhel and V. Strigalev, "Method for eliminating the noise signal influence on the sensitivity of receiving hydroacoustic antenna based on fiber bragg gratings," Scientific and Technical Journal of Information Technologies, Mechanics and Optics, no. 5(69), pp. 5-9, 2010.

[10] Y. Liu, L. Wang, C. Tian, M. Zhang, and Y. Liao, "Analysis and optimization of the PGC method in all digital demodulation systems," Journal of Lightwave Technology, vol. 26, no. 18, pp. 3225-3233, 2008.

[11] L. Feng, J. He, J.-Y. Duan, F. Li, and Y.-L. Liu, "Implementation of phase generated carrier technique for FBG laser sensor multiplexed system based on Compact RIO," Journal of Electronic Science and Technology of China, vol. 6, no. 4, pp. 385-388, 2008.

[12] A. I. Azmi, I. Leung, X. Chen et al., "Fiber laser based hydrophone systems," Photonic Sensors, vol. 1, no. 3, pp. 210-221, 2011.

[13] Y. Zhang, N. Wang, X. Li, and X. Sun, "Study of PGC demodulation scheme for interferometric fiber-optic sensors," in Proceedings of the Symposium on Photonics and Optoelectronics, pp. 1-4, Shanghai, China, 2012. 

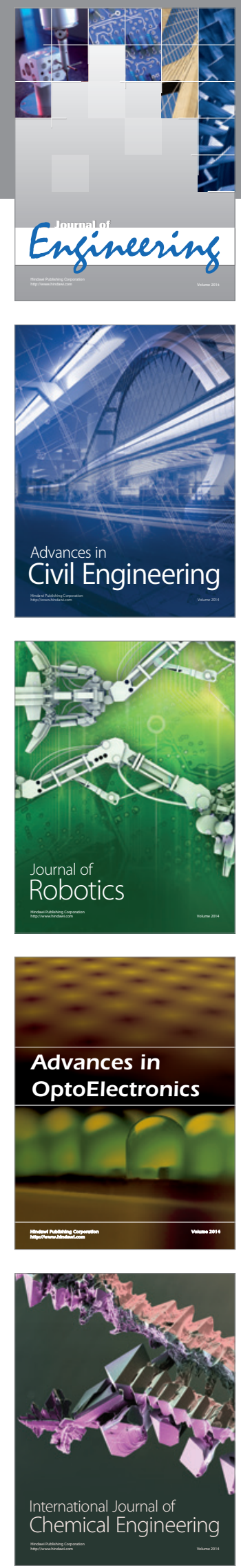

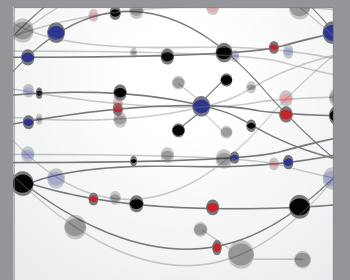

The Scientific World Journal
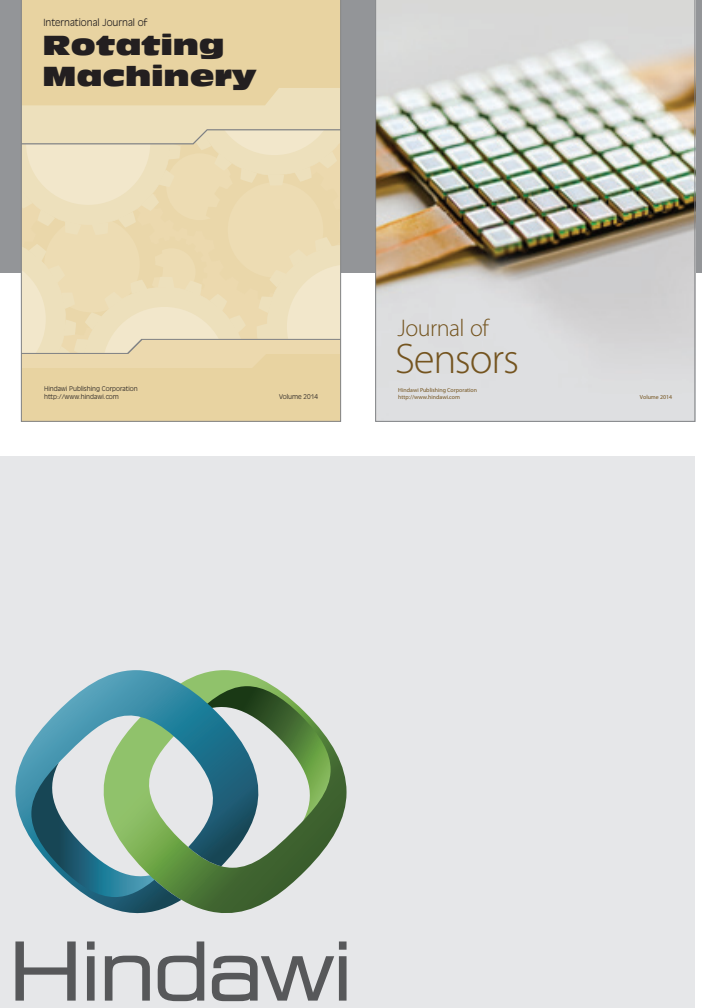

Submit your manuscripts at http://www.hindawi.com
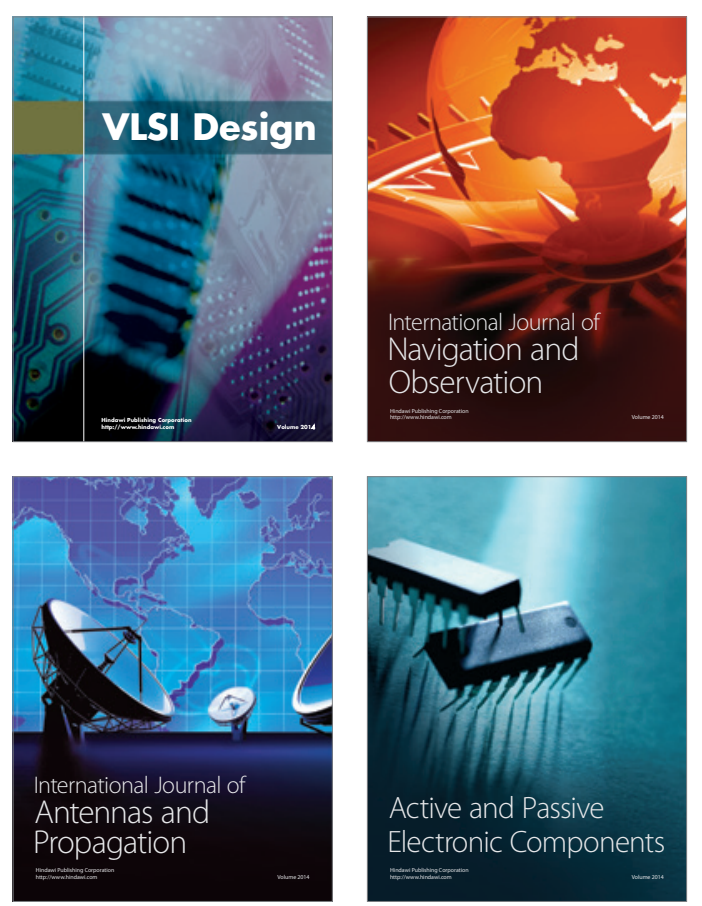
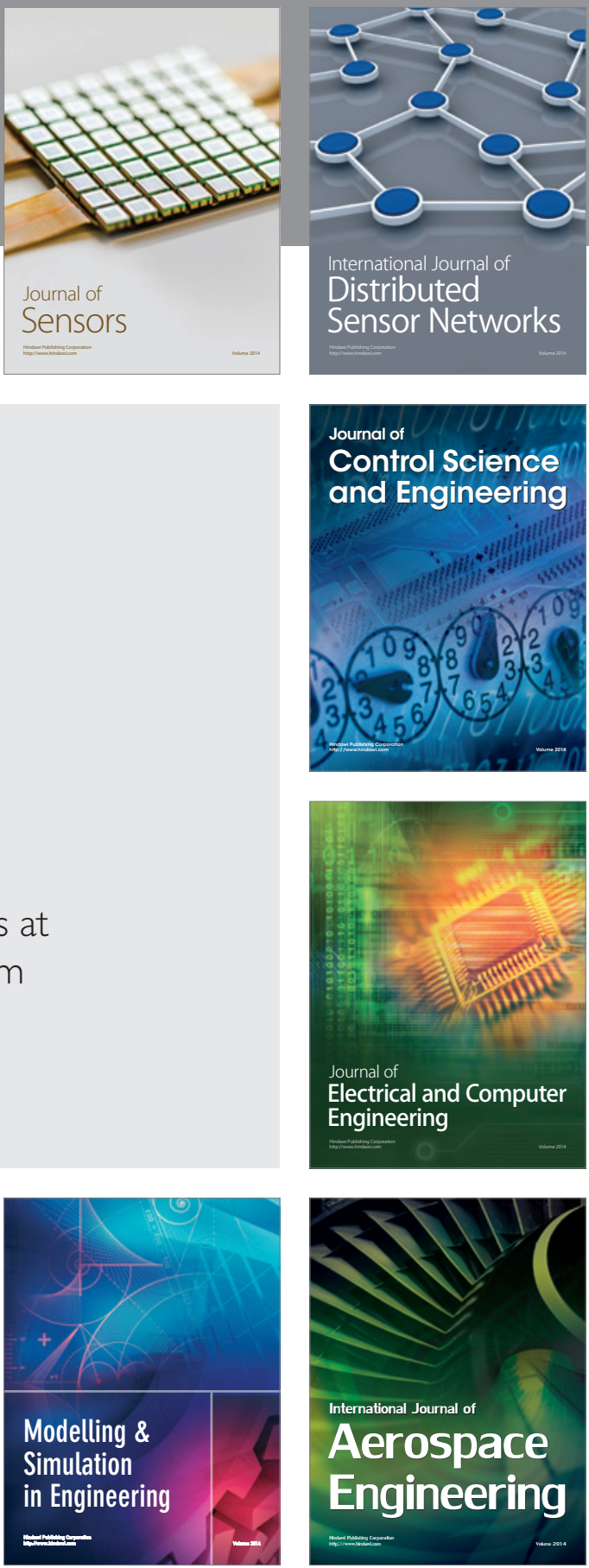

Journal of

Control Science

and Engineering
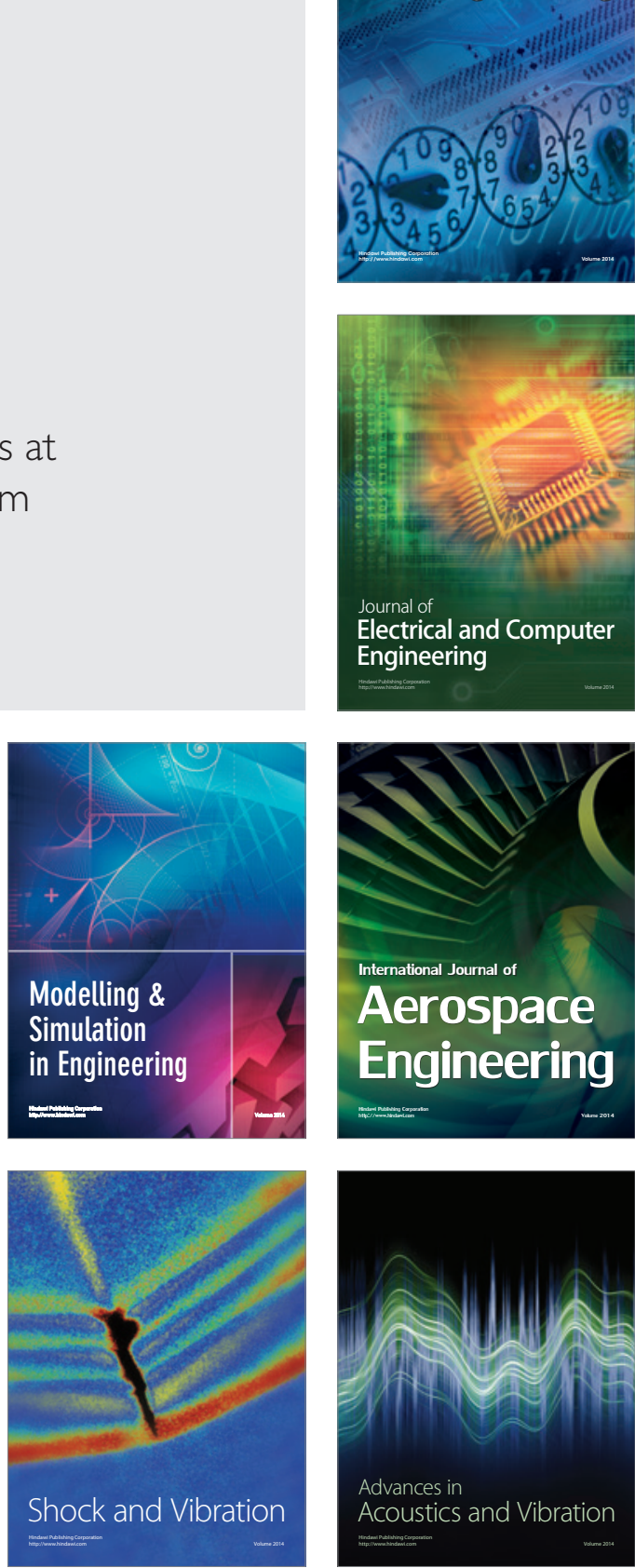